\title{
A Visual Analytics Dashboard to Summarize Serial Anesthesia Records in Pediatric Radiation Treatment
}

\author{
Olivia Nelson ${ }^{1}$ Brian Sturgis ${ }^{2}$ Keri Gilbert ${ }^{1} \quad$ Elizabeth Henry $^{3} \quad$ Kelly Clegg ${ }^{3}$ Jonathan M. Tan ${ }^{1,4}$ \\ Jack O. Wasey ${ }^{1}$ Allan F. Simpao ${ }^{1,4}$ Jorge A. Gálvez ${ }^{1,4}$
}

${ }^{1}$ Department of Anesthesiology and Critical Care Medicine, Children's Hospital of Philadelphia, Philadelphia, Pennsylvania, United States

2 Enterprise Reporting \& Analytics, Children's Hospital of Philadelphia, Philadelphia, Pennsylvania, United States

3 Pediatric Proton Therapy Center, Perelman Center for Advanced Medicine, Children's Hospital of Philadelphia, Philadelphia, Pennsylvania, United States

${ }^{4}$ Department of Anesthesiology and Critical Care, Perelman School of Medicine at the University of Pennsylvania, Philadelphia,

Pennsylvania, United States

Appl Clin Inform 2019;10:563-569.
Address for correspondence Jorge A. Gálvez, MD, MBI, Department of Anesthesiology and Critical Care, Perelman School of Medicine at the University of Pennsylvania, 34th Street \& Civic Center Boulevard, Philadelphia, PA 19104-4399, United States

(e-mail: galvezj@email.chop.edu).

\section{Abstract}

Keywords

- data warehousing

- documentation

- efficiency

- electronic health records

- workflow
Background Young children who undergo radiation therapy may require general anesthesia to remain still during weeks of radiation sessions. On a typical day at our hospital, an anesthesia team will care for 10 patients in the radiation therapy suite, and each patient will have multiple prior anesthetic records. Daily review of prior anesthesia records is important to maintain anesthetic consistency and to identify potential improvement, yet our electronic health record (EHR) made such review time-consuming and cumbersome.

Objectives This article aims to design a visual analytics interface that simultaneously displays data from multiple anesthesia encounters to support clinical consistency in medications and airway management.

Methods Documentation from the EHR is available in the clinical data warehouse following daily backups. A visual analytics interface was built to aggregate important components of multiple anesthesia encounters in pediatric radiation oncology on a single screen. The application was embedded in the EHR's anesthesia module and updated daily. Results Each anesthesia encounter was represented by a vertical line with the date at the bottom of the screen. Each vertical line was divided into sections corresponding to the medications, type of airway device, type of radiation oncology procedure, days between treatments, and recovery score and time. Information about the medications, airways, and procedures was shown with icon legends. This layout enabled users to quickly see the key components of multiple anesthetics and make inferences between, for example, the medications used and the recovery score.

Conclusion The dashboard provides a high-level summary of all radiation therapy anesthesia records for children receiving recurrent treatments. In this clinical scenario, it is desirable to replicate an optimal anesthetic approach for daily or near-daily treatments or adjust the anesthetic based on observed patterns. received

April 15, 2019

accepted after revision June 17, 2019
(C) 2019 Georg Thieme Verlag KG Stuttgart · New York
DOI https://doi.org/

$10.1055 / \mathrm{s}-0039-1693712$ ISSN 1869-0327. 


\section{Background and Significance}

Children undergoing radiation therapy require sedation or general anesthesia to enable them to lie still during their treatments. ${ }^{1}$ Radiation sessions are scheduled on a daily or near-daily basis for treatment courses that typically extend over several weeks. At our institution, the optimal anesthetic medication doses and infusion rates for each patient are often determined early in their treatment courses. Patients with head and neck lesions receive a customized mask to replicate the same position over multiple treatments, and the profile of the airway device that is used during general anesthesia forms part of the mask shape. ${ }^{2}$ Prior work on a prototype of the dashboard by our group found an association between changes in the type of airway device and an unscheduled proton therapy simulation session to allow fitting of a new customized mask. This was accompanied by a change in treatment from proton therapy to conventional radiation therapy during planning of proton therapy with the new mask shape. ${ }^{3}$ Thus, the anesthesia team strives to maintain consistency with each patient's anesthetic, and on the morning of a treatment day, the anesthesia, nursing, and radiation oncology teams meet to discuss each patient's recent anesthetics and the anesthesia plan for the day.

Unfortunately, viewing each prior anesthetic record in our electronic health record (EHR) requires excessive scrolling and multiple clicks, thereby hindering quick, efficient review of weeks or months of anesthesia records. Thus, we convened a focus group of anesthesiologists, nurse anesthetists, and radiation oncology nurses to discuss the relevant elements of the anesthesia record. These were determined to be the medications, the airway device, and the recovery time and quality. To provide a broader view of the patient's clinical radiation course, we also included the days between treatments and the type of procedure. We then built a visual analytics tool to display this key data from multiple anesthesia encounters in a single screen. The resulting dashboard was created in accordance with the design heuristic principles of match between system and the real world and aesthetic and minimal design. ${ }^{4,5}$ Specifically, we used visually distinct colors for the different sections of the dashboard and used icons rather than text where possible. Data elements in the dashboard are placed in locations similar to the existing anesthesia record system, and data are displayed in a left to right chronological orientation. The visual analytics interface enables focused review of multiple prior anesthetics and allows the anesthesia team to visualize patterns between the anesthetic management and the time and quality of recovery in a patient receiving multiple similar anesthetics within a short time period.

Initial assessment of the usability of the dashboard was based on prior usability studies that quantified the number of clicks required to complete common EHR orders ${ }^{6}$ and involved quantifying user input device interactions while using the dashboard versus the EHR. We counted the number of mouse button clicks needed to obtain the same information from the dashboard and prior anesthetic records. To account for usage differences between the prior anesthetic records review and the visual analytics dashboard, we counted the number of mouse scroll clicks needed to review the multiple pages of prior anesthesia records and the number of mouse hovers on the dashboard view to see all doses of one medication type.

\section{Objectives}

Our goal was to develop and implement a visual analytics interface that simultaneously displays key data from multiple anesthesia encounters in a single screen to facilitate review of prior anesthetics and to visualize patterns between anesthetic management and patient recovery characteristics.

\section{Methods}

Clinical documentation is conducted in the EHR(Epic Systems, Verona, Wisconsin, United States). The clinical data that is backed up to a clinical data warehouse every 24 hours is available for reporting applications (-Fig. 1). A visual analytics interface (Qlikview, QlikTech, Radnor, Pennsylvania, United States) was built to aggregate data from all anesthesia encounters in pediatric radiation oncology at the Children's Hospital of Philadelphia. Patients were included if they received general anesthesia in the radiation oncology suite at the Children's Hospital of Philadelphia. Anesthetic records from other units in the hospital were not included.

The display includes the patient schedule, medications administered, airway device used, radiation procedure completed, recovery room time, and agitation scale using the Watcha score. ${ }^{7}$ The dashboard application was embedded in the EHR's anesthesia module and made accessible to clinicians in the pediatric radiation therapy suite. The application was set to update at 6:00 a.m. daily.

Usability testing of the dashboard was conducted and compared with the standard EHR interface. The number of clicks and mouse scroll clicks to go through the multiple screens of a single anesthesia record on our existing EHR were counted by a nurse anesthetist and reviewed by a senior attending anesthesiologist (J.A.G.). The same process was repeated using the patient visual analytics dashboard. Click and scroll click counts for each method were then calculated using the number of patients on the actual schedule and the number of prior anesthetic records for each of these patients on a randomly selected clinical day. The number of mouse hovers required to review the dosage of one medication per prior anesthetic record was assessed for the dashboard view.

\section{Results}

The Qlikview application runs on the hospital server and is available as a hyperlink within the EHR. The dashboard displays a list of the patients scheduled to receive anesthesia for radiation therapy on the left side of the screen (-Fig. 2). The dashboard display is activated by clicking on each patient. The patient-level view displays each anesthesia encounter as a vertical line with the date at the bottom of the screen; serial anesthetics are shown sequentially from left to right. Each vertical line was divided into four sections 


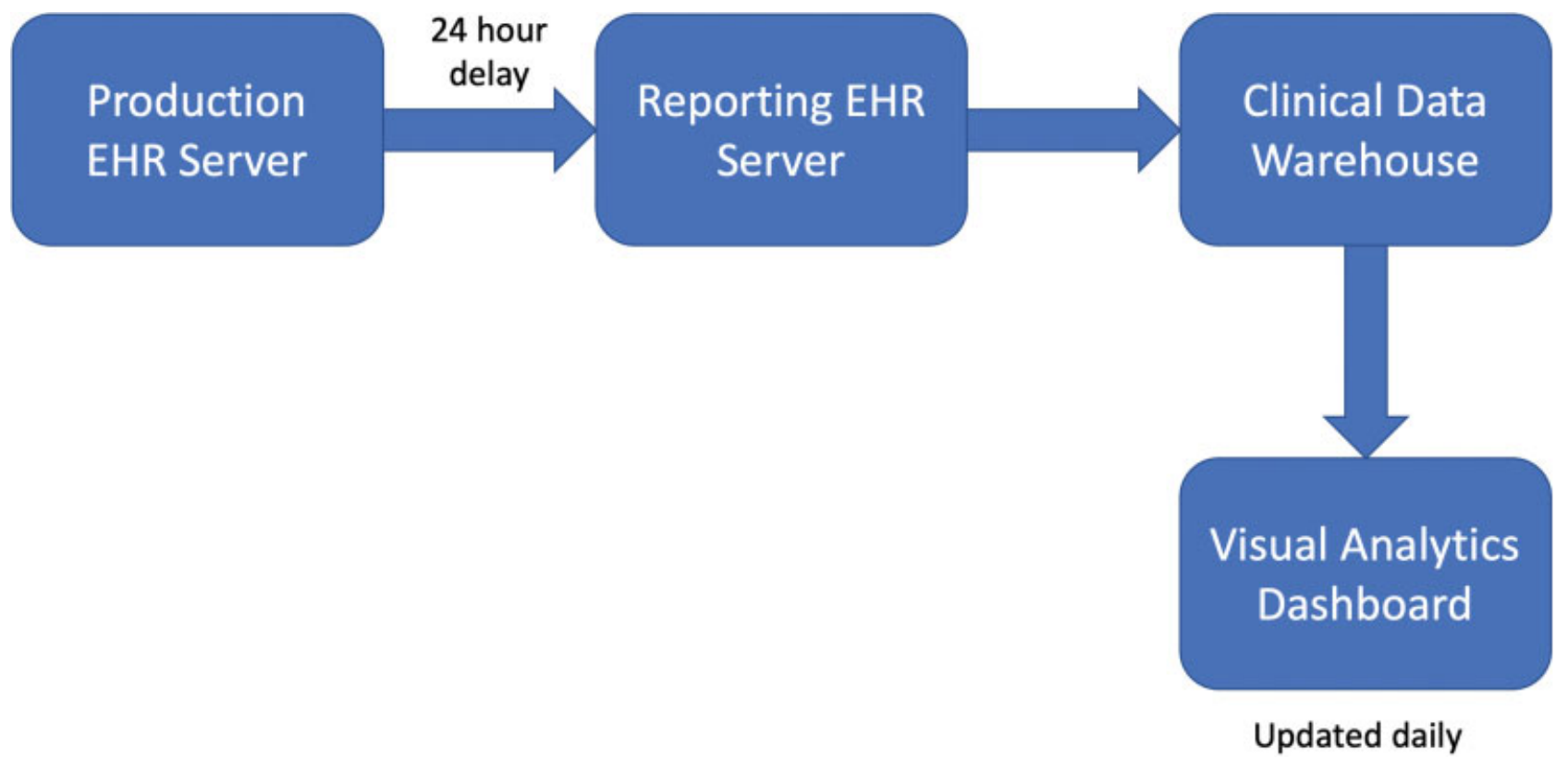

Fig. 1 The production electronic health record (EHR) is backed up to the reporting EHR server with a 24-hour delay. Once documentation is in the EHR server, it is available to the clinical data warehouse (CDW). Data in the CDW is then exported to the visual analytics dashboard daily in the early morning.
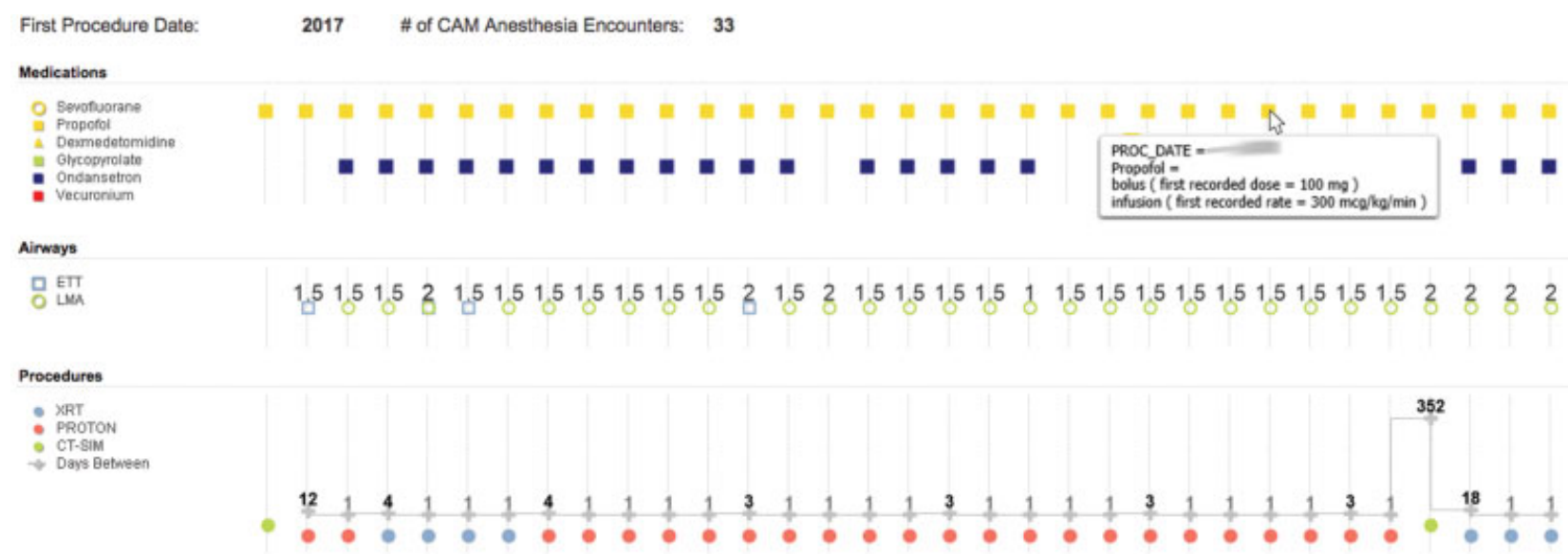

Recovery Score \& Time

- Time to Phase ?

A Delitium Scale

$\begin{array}{llllllllllllllllllllllllllll}1 & 1 & 1 & 1 & 1 & 1 & 1 & 1 & 1 & 1 & 1 & 1 & 1 & 1 & 1 & 1 & 1 & 1 & 1 & 1 & 1\end{array}$

12

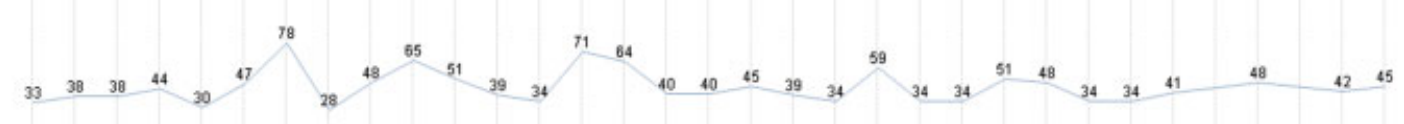

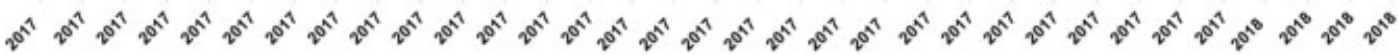

Fig. 2 The dashboard is divided into four sections with icon legends: medications, airways, procedures, and recovery score and time. Each anesthetic is represented by a vertical line chronologically from left to right, with the date of each encounter at the bottom of the screen (exact dates removed here). The Medications section includes those commonly used for this type of general anesthetic, with separate icons for sevoflurane, propofol, dexmedetomidine, glycopyrrolate, ondansetron, and vecuronium. Dosage is shown if the mouse cursor is hovered over an icon, as shown by the cursor. The Airway section shows icons for endotracheal tubes (ETTs) or laryngeal mask airways (LMAs) as well as a number corresponding to the size of the device. The Procedures section shows common procedure types performed in pediatric radiation therapy, including computed tomography (CT) simulation (CT-SIM), conventional radiation therapy (XRT), and proton radiation therapy (PROTON). Above the Procedures section, the number of days between treatments is listed to assist with identifying gaps in treatment. The Recovery Score shows the maximum recovery score based on the Watcha scale (1-calm, asleep, 2-calm, can be consoled, 3-crying, cannot be consoled, 4-thrashing and inconsolable). The Recovery Time in minutes is listed above the line representing the Recovery Scale. 
with icon legends: medications, airways, procedures, and recovery score and time.

\section{Medication}

The medication icons display the dose administered for each agent (e.g., propofol bolus, propofol infusion rate, ondansetron) when the mouse cursor hovers over the respective medication icon.

\section{Airway}

The airway section shows icons for endotracheal tube (ETT) or laryngeal mask airway (LMA) and a number to denote the size of the device (e.g., a green circle with 1.5 represents a size 1.5 LMA).

\section{Procedure}

The procedures section shows the procedure completed during each anesthesia encounter, including computed tomography simulation (CT-SIM), conventional radiation therapy (XRT), and proton radiation therapy (PROTON). Of note, if a patient receives general anesthesia but does not complete a treatment procedure (i.e., proton therapy cancelled), then the procedure icon is blank. The "days between" field represents the number of days between the current and previous anesthetic; this field is useful in identifying gaps in treatment. For example, the patient displayed in -Fig. 2 underwent one course of radiation therapy that ended in 2017, and then began another course of radiation therapy 352 days later. Most radiation therapy patients undergo a series of multiple treatments over a span of weeks to months.

\section{Recovery}

The recovery section includes "Time to Phase 2" representing the duration of the initial postanesthesia recovery phase in minutes. The delirium scale shows the patient's maximum recovery score based on the Watcha scale (1-calm, asleep, 2-calm, can be consoled, 3-crying, cannot be consoled, 4-thrashing and inconsolable). ${ }^{7}$ A summary view enables users to identify recovery patterns and changes across multiple anesthetics more easily than the onerous native
EHR interface. For example, in the dashboard, the medications given and their dosages for each anesthetic are displayed along with the corresponding recovery time and score. In the native EHR interface, viewing the same information would involve accessing each anesthetic record individually.

Usability testing found that the patient dashboard required three clicks to open to the screen showing the summary view of all of the prior anesthetics for a patient ( - Table 1). Each prior anesthetic also required approximately one mouse hover per medication icon to review the relevant intravenous anesthetic dosage given. In contrast, the existing EHR required three clicks to access the anesthesia section of the chart, followed by additional clicks and mouse scroll clicks to view the approximately 14.5 screens that comprise each prior anesthetic record. Reviewing each additional prior anesthetic record required 3 clicks and 109 mouse scroll clicks. There were 5 patients on the schedule, each with several prior anesthetic records, ranging from 17 to 32 . Review of all prior anesthetic records using the traditional EHR would require 235 clicks and 12,535 mouse scroll clicks to see 1,677.5 screens of data.

The summary view of multiple anesthetics can also facilitate the identification of documentation errors in the medical record. For example, in the case displayed in - Fig. 2, both ETTs and LMAs were documented, but the ETT size of 2.0 corresponds to the correct size 2 LMA for this patient. A 2.0 ETT would be far too small for this patient. A documentation error involving the wrong dose of ondansetron documented in the record (12 mg instead of $1.2 \mathrm{mg}$ ) was also revealed by the dashboard format. Inconsistencies in medication dosing are more easily seen on the dashboard view.

The utility of this visual analytics tool is not limited to the anesthesia team. The radiation therapy team can use the visual dashboard to review the plan for each patient. The radiation therapy multidisciplinary team at our institution has a daily morning meeting to discuss the plan for each patient. The dashboard serves as a visual tool to improve situational awareness of the anesthetic plan and potential issues for the entire radiation oncology team.

Table 1 Prior to starting the click counting, the computer was on, Epic was logged on under the anesthesia profile, and was showing the "My Cases" status board

\begin{tabular}{|c|c|c|c|c|c|c|c|}
\hline \multirow[t]{2}{*}{ Patient } & \multirow{2}{*}{$\begin{array}{l}\text { Number of } \\
\text { anesthetic } \\
\text { records }\end{array}$} & \multicolumn{3}{|l|}{ EHR } & \multicolumn{3}{|l|}{ Visual dashboard } \\
\hline & & $\begin{array}{l}\text { Number of clicks } \\
\text { to open all } \\
\text { prior records }\end{array}$ & $\begin{array}{l}\text { Number of } \\
\text { screen pages } \\
\text { in prior records }\end{array}$ & $\begin{array}{l}\text { Number of } \\
\text { mouse } \\
\text { scroll clicks }\end{array}$ & $\begin{array}{l}\text { Number of } \\
\text { clicks to review } \\
\text { prior records }\end{array}$ & $\begin{array}{l}\text { Number of } \\
\text { screen pages }\end{array}$ & $\begin{array}{l}\text { Number of } \\
\text { mouse } \\
\text { hovers }\end{array}$ \\
\hline 1 & 23 & 47 & 333.5 & 2,507 & 3 & 1 & 23 \\
\hline 2 & 17 & 35 & 246.5 & 1,853 & 3 & 1 & 17 \\
\hline 3 & 17 & 35 & 246.5 & 1,853 & 3 & 1 & 17 \\
\hline 4 & 32 & 65 & 464 & 3,488 & 3 & 1 & 32 \\
\hline 5 & 26 & 53 & 377 & 2,834 & 3 & 1 & 26 \\
\hline Total & 115 & 235 & 1667.5 & 12,535 & 15 & 5 & 115 \\
\hline
\end{tabular}

Abbreviation: EHR, electronic health record.

Note: All clicks were counted from the 'my cases' status board in Epic. 


\section{Discussion}

We successfully designed and implemented a visual analytics tool that automatically refreshes each morning with an updated, graphical display of multiple prior anesthetics that allows the anesthesia team to optimize the care of radiation therapy patients and visualize patterns in anesthetic management and patient recovery. The dashboard facilitates discussion during the radiation oncology suite's multidisciplinary daily morning meeting.

The dashboard provides a high-level summary of all radiation therapy anesthesia records for children receiving recurrent treatments. In this clinical setting, it is desirable to replicate an optimal anesthetic approach each day or to adjust the anesthetic based on observed patterns. Inconsistencies in medication dosing and recovery time from day to day are more readily reviewed in the dashboard format. Patients who undergo radiation treatments have minimal time between anesthetics, which allowed us to select key elements of the anesthetic record for review in a single screen visual analytics format. We then incorporated this tool into our daily pediatric radiation oncology meetings and used it to assist with formulating the anesthetic plan for each patient. The overview of key anesthetic elements also highlighted airway documentation errors and prompted us to redesign our airway documentation interface in the EHR.

The dashboard's unanticipated utility in identifying documentation errors has provided significant benefits. For example, the airway documentation error mentioned in the "Results" ultimately led to the redesign of the EHR user interface for airway device documentation for better workflow integration and to reduce user data entry errors.

Data visualization through visual analytical platforms incorporates concepts from human factors engineering and cognitive psychology. Information visualization can decrease information overload ${ }^{8,9}$ and has been shown to improve recall of important clinical information. ${ }^{10}$ High-dimensional data can support clinical decision making. ${ }^{8}$ Visual analytics dashboards have been used to allow real-time tracking of information in health care, including creation of a hospitalspecific antibiogram, ${ }^{11}$ to monitor for adverse drug events, ${ }^{12}$ and to track departmental performance metrics. ${ }^{13}$

Visual analytics tools have been used across various health care settings to decrease information overload. The Glucolyzer is a visual analytics tool that utilizes patientgenerated data to help dieticians see links between dietary intake and subsequent blood glucose in patients with type 2 diabetes. Dieticians using the Glucolyzer tool as compared with a standard diet log book reported decreased information overload. Interestingly, this visual analytics overview of multiple blood glucose and diet parings illuminated recurring patterns such that these dieticians were able to make more complex connections between dietary intake and subsequent blood glucose. ${ }^{14}$ In this case, subtracting superfluous information allowed clinicians to draw connections between relevant data. Dashboards have also been used to provide clinicians caring for patients with diabetes with decision support based on longitudinal predictive modeling.
Clinicians who used such a dashboard had decreased visit times and increased screening rates for complications of diabetes. ${ }^{15}$ Implementation of a dashboard in the surgical intensive care unit increased compliance with a ventilatorassociated pneumonia (VAP) prevention care bundle and decreased rates of VAP. ${ }^{16}$ The VAP compliance dashboard is similar to many other dashboards in that it employs a redyellow-green traffic light motif as a visual short cut to convey areas of concern. ${ }^{17}$

Prior information visualization work in the anesthesia environment has centered around decreasing information overload and increasing situational awareness of the multiple streams of physiologic and patient data that are available to anesthesiologists in the operating room..$^{10,18-21}$ Few applications of visual analytics to anesthesia data to facilitate preoperative processes have been described in the literature. Visual analytics has been used to explore perioperative blood transfusion patterns to guide preoperative blood ordering practices. ${ }^{22} \mathrm{~A}$ visual analytics approach has been used to analyze nonanesthesiologists' utilization of preoperative assessments. ${ }^{23}$ Our dashboard is a novel application of visual analytics that complements these earlier applications and gives clinicians a second, potentially more efficient, option to review prior anesthetic data in the preoperative period.

The dashboard's unanticipated utility in identifying documentation errors has provided significant benefits. For example, the airway documentation error mentioned in the "Results" ultimately led to the redesign of the EHR user interface for airway device documentation for better workflow integration and to reduce user data entry errors.

\section{Conclusion}

The primary goal of this project was to provide information on prior anesthetics to allow clinicians to tailor their anesthetic plan based on a more complete understanding of prior anesthetic experiences for each patient. The patient visual dashboard drastically decreased the number of clicks and mouse scroll clicks needed to review prior anesthetic records. In the future, we will continue to further assess and refine this prototype based on feedback from anesthesiologists and other members of the pediatric radiation oncology team and to develop similar visual analytics dashboards to summarize multiple anesthesia records for other patient populations.

\section{Clinical Relevance Statement}

The visual analytics dashboard describes an innovative solution to the clinical problem of inefficient patient records review in EHR systems in which patient data are spread over multiple screens and records review is a click-intensive process. We created a single screen view of key components of serial anesthetics for patients undergoing daily or near-daily anesthesia to allow efficient records review and comparison of recovery trends. This type of summary dashboard can be utilized for multiple clinical areas in which patients are undergoing serial anesthetics. 


\section{Multiple Choice Questions}

1. The pediatric radiation therapy anesthesia visual analytics dashboard can be used to identify which of the following?

a. Whether recovery scores fall within accepted optimal standards as shown by red-yellow-green denotations.

b. Predict which patients would benefit from additional antiemetic medication.

c. See qualitative trends between individual medication doses and recovery quality and duration.

d. Identify patients at risk of cancer relapse.

Correct Answer: The correct answer is option c. The dashboard provides a distilled view of an individual patient's prior anesthetic records. As such, it can be used to see trends between medication doses and recovery times as well as the quality of recovery. Standards and benchmarking for optimal anesthetic recovery from pediatric radiation therapy have not yet been created (a). Although the dashboard would show which patients have received multiple antiemetic medications in the past, it does not provide the granularity of data to show postanesthetic nausea and vomiting. However, a prolonged recovery and/or the medication history might prompt the anesthesiologist to inquire about this issue (b). The dashboard provides anesthetic information; while it would show gaps in treatment or changes in treatment type from proton to conventional radiation therapy, it would not assist with predicting response to radiation treatment $(\mathrm{d})$.

2. Why is proton radiation therapy preferred in pediatric head and neck malignancies?

a. Despite wider margins of healthy tissue damage, the effectiveness is higher.

b. Patient positioning can be less precise, and therefore treatment gaps are uncommon.

c. The cost is lower and it is widely available at several centers.

d. The proton beam does not have an exit path and nearby tissue receives less radiation.

Correct Answer: The correct answer is option d. Proton therapy is preferred in pediatric patients because it is more precisely targeted to the tumor. The beam does not go past the tumor; therefore, it does not damage the healthy tissue that is located beyond the tumor $(\mathrm{d}, \mathrm{a})$. This targeting comes at the cost of requiring careful replication of patient position, and changes in patient position can cause gaps in treatment (b). Although proton therapy is offered by an increasing number of centers, it is still less accessible than conventional radiation therapy (c).

\section{Protection of Human and Animal Subjects}

The study was performed in compliance with the World Medical Association Declaration of Helsinki on Ethical Principles for Medical Research Involving Human Subjects. The study was reviewed by the Institutional Review
Board of the Children's Hospital of Philadelphia and determined not to be human subjects research.

Conflict of Interest

None declared.

\section{References}

1 McFadyen JG, Pelly N, Orr RJ. Sedation and anesthesia for the pediatric patient undergoing radiation therapy. Curr Opin Anaesthesiol 2011;24(04):433-438

2 Vogel J, Both S, Kirk M, et al. Proton therapy for pediatric head and neck malignancies. Pediatr Blood Cancer 2018;65(02):e26858

3 Chua P, Hill-Kayser C, Ahumada LM, et al. Visual analytics dashboard to explore the relationship of unscheduled treatment interruptions and variations in airway management for children undergoing external beam radiation therapy. Pract Radiat Oncol 2017;7(05):e339-e344

4 Miller K, Capan M, Weldon D, et al. The design of decisions: matching clinical decision support recommendations to Nielsen's design heuristics. Int J Med Inform 2018;117:19-25

5 Dowding D, Merrill JA. The development of heuristics for evaluation of dashboard visualizations. Appl Clin Inform 2018;9(03): 511-518

6 Ratwani RM, Savage E, Will A, et al. A usability and safety analysis of electronic health records: a multi-center study. J Am Med Inform Assoc 2018;25(09):1197-1201

7 Watcha MF, Ramirez-Ruiz M, White PF, Jones MB, Lagueruela RG, Terkonda RP. Perioperative effects of oral ketorolac and acetaminophen in children undergoing bilateral myringotomy. Can J Anaesth 1992;39(07):649-654

8 Simpao AF, Ahumada LM, Gálvez JA, Rehman MA. A review of analytics and clinical informatics in health care. J Med Syst 2014; 38(04):45

9 Caban JJ, Gotz D. Visual analytics in healthcare-opportunities and research challenges. J Am Med Inform Assoc 2015;22(02):260-262

10 Tscholl DW, Handschin L, Neubauer P, et al. Using an animated patient avatar to improve perception of vital sign information by anaesthesia professionals. Br J Anaesth 2018;121(03):662-671

11 Simpao AF, Ahumada LM, Larru Martinez B, et al. Design and implementation of a visual analytics electronic antibiogram within an electronic health record system at a tertiary pediatric hospital. Appl Clin Inform 2018;9(01):37-45

12 Sorbello A, Ripple A, Tonning J, et al. Harnessing scientific literature reports for pharmacovigilance. Prototype software analytical tool development and usability testing. Appl Clin Inform 2017;8 (01):291-305

13 Karami M, Safdari R. From information management to information visualization: development of radiology dashboards. Appl Clin Inform 2016;7(02):308-329

14 Feller DJ, Burgermaster M, Levine ME, et al. A visual analytics approach for pattern-recognition in patient-generated data. J Am Med Inform Assoc 2018;25(10):1366-1374

15 Dagliati A, Sacchi L, Tibollo V, et al. A dashboard-based system for supporting diabetes care. J Am Med Inform Assoc 2018;25(05): $538-547$

16 Zaydfudim V, Dossett LA, Starmer JM, et al. Implementation of a real-time compliance dashboard to help reduce SICU ventilatorassociated pneumonia with the ventilator bundle. Arch Surg 2009;144(07):656-662

17 Dowding D, Randell R, Gardner P, et al. Dashboards for improving patient care: review of the literature. Int J Med Inform 2015;84 (02):87-100

18 Nair BG, Gabel E, Hofer I, Schwid HA, Cannesson M. Intraoperative clinical decision support for anesthesia: a narrative review of available systems. Anesth Analg 2017;124(02):603-617 
19 Freundlich RE, Ehrenfeld JM. Anesthesia information management: clinical decision support. Curr Opin Anaesthesiol 2017;30 (06):705-709

20 Simpao AF, Tan JM, Lingappan AM, Gálvez JA, Morgan SE, Krall MA. A systematic review of near real-time and point-of-care clinical decision support in anesthesia information management systems. J Clin Monit Comput 2017;31(05):885-894

21 Tremper KK, Mace JJ, Gombert JM, Tremper TT, Adams JF, Bagian JP. Design of a novel multifunction decision support display for anesthesia care: AlertWatch OR. BMC Anesthesiol 2018;18(01):16

22 Gálvez JA, Ahumada L, Simpao AF, et al. Visual analytical tool for evaluation of 10 -year perioperative transfusion practice at a children's hospital. J Am Med Inform Assoc 2014;21(03): 529-534

23 Wanderer JP, Gruss CL, Ehrenfeld JM. Using visual analytics to determine the utilization of preoperative anesthesia assessment. Appl Clin Inform 2015;6(04):629-637 